\title{
ARTICLE
}

\section{The association between lithium use and neurocognitive performance in patients with bipolar disorder}

Katherine E. Burdick ${ }^{1,2,3}$, Caitlin E. Millett ${ }^{1,2}$, Manuela Russo ${ }^{4}$, Martin Alda ${ }^{5}{ }^{5}$, Ney Alliey-Rodriguez ${ }^{6}$, Amit Anand ${ }^{7}$, Yokesh Balaraman ${ }^{7}$, Wade Berrettini ${ }^{8}$, Holli Bertram (D) $^{9}$, Joseph R. Calabrese (D) $^{10}$, Cynthia Calkin iD $^{5}$, Carla Conroy ${ }^{10}$, William Coryell ${ }^{11}$, Anna DeModena ${ }^{12}$, Scott Feeder $^{13}$, Carrie Fisher ${ }^{7}$, Nicole Frazier ${ }^{9}$, Mark Frye ${ }^{13}$, Keming Gao ${ }^{10}$, Julie Garnham (iD ${ }^{5}$, Elliot S. Gershon (D) ${ }^{6}$, Kara Glazer ${ }^{14}$, Fernando S. Goes $\mathbb{D}^{14}{ }^{14}$, Toyomi Goto ${ }^{10}$, Gloria J. Harrington ${ }^{9}$, Petter Jakobsen ${ }^{15}$, Masoud Kamali ${ }^{9}$, Marisa Kelly (D) $^{9}$, Susan Leckband ${ }^{12}$, Else Marie Løberg ${ }^{15,16,17}$, Falk W. Lohoff ${ }^{18}$, Adam X. Maihofer $\mathbb{D}^{12}$, Michael J. McCarthy $\mathbb{D}^{12}$, Melvin Mclnnis $\mathbb{D}^{9}$, Gunnar Morken $\mathbb{D}^{19}$, Caroline M. Nievergelt $\mathbb{i D}^{12}$, John Nurnberger ${ }^{7}$, Ketil J. Oedegaard ${ }^{15}$, Abigail Ortiz $\mathbb{D D}^{20}$, Megan Ritchey ${ }^{14}$, Kelly Ryan ${ }^{9}$, Martha Schinagle ${ }^{10}$, Candice Schwebel $^{8}$, Martha Shaw ${ }^{11}$, Paul Shilling ${ }^{12}$, Claire Slaney ${ }^{5}$ Emma Stapp (iD ${ }^{14}$, Bruce Tarwater $^{11}$, Peter Zandi ${ }^{14}$ and John R. Kelsoe (iD) ${ }^{12}$

Lithium remains the gold standard for the treatment of bipolar disorder (BD); however, its use has declined over the years mainly due to the side effects and the subjective experience of cognitive numbness reported by patients. In the present study, we aim to methodically test the effects of lithium on neurocognitive functioning in the largest single cohort $(n=262)$ of BD patients reported to date by harnessing the power of a multi-site, ongoing clinical trial of lithium monotherapy. At the cross-sectional level, multivariate analysis of covariance (MANCOVA) was conducted to examine potential group differences across neurocognitive tests [California Verbal Learning Test (CVLT trials 1-5,CVLT delayed recall), Wechsler Digit Symbol, Trail-making Test parts A and B (TMT-A; TMT-B), and a global cognition index]. At the longitudinal level, on a subset of patients $(n=88)$ who achieved mood stabilization with lithium monotherapy, we explored the effect of lithium treatment across time on neurocognitive functioning. There were no differences at baseline between BD patients that were taking lithium compared with those that were not. At follow-up a significant neurocognitive improvement in the global cognitive index score $[F=31.69 ; p<0.001]$, CVLT trials $1-5[F=29.81 ; p<0.001]$, CVLT delayed recall $[F=15.27 ; p<0.001]$, and TMT-B $[F=6.64, p=0.012]$ was detected. The cross-sectional and longitudinal (on a subset of 88 patients) investigations suggest that lithium may be beneficial to neurocognitive functioning in patients with $B D$ and that at the very least it does not seem to significantly impair cognition when used therapeutically.

Neuropsychopharmacology (2020) 45:1743-1749; https://doi.org/10.1038/s41386-020-0683-2

\section{INTRODUCTION}

Neurocognitive impairment is common in patients with bipolar disorder (BD), even during periods of affective remission [1]. Persistent deficits in attention, verbal learning/memory, and some executive functions [2] have been directly linked to functional disability [3, 4], making these deficits particularly relevant to patients' quality of life. The nature of these impairments is not well understood, with recent data suggesting significant heterogeneity in outcome [5]. Early evidence suggests that cognitive impairments in BD are caused by a number of factors, stemming both from abnormal early development/genetic predisposition [6, 7], medical/cardiovascular comorbidities [8, 9], and from neurodegenerative processes [10] related to the expression of the disorder and/or to its treatment. The potential adverse effects of psychotropic medications have long been described anecdotally but generally have not been systematically characterized. This is of particular significance as it is one of the more modifiable aspects contributing to reported cognitive difficulties in patients with BD.

Among the plethora of psychotropic agents now available to treat differing aspects of $\mathrm{BD}$, lithium has been in use the longest and it remains a first-line treatment option because of its efficacy in long-term prophylaxis and its positive effects on suicide risk $[11,12]$. Despite a number of potentially serious adverse effects on renal and endocrine function [13], patients frequently complain of weight gain and a subjective experience of neurocognitive dulling $[14,15]$. Importantly, BD patients list cognitive dysfunction,

\footnotetext{
${ }^{1}$ Department of Psychiatry, Brigham and Women's Hospital, Boston, MA, USA; ${ }^{2}$ Department of Psychiatry, Harvard Medical School, Boston, MA, USA; ${ }^{3}$ ames J Peters Veteran Administration (VA) Hospital, Bronx, NY, USA; ${ }^{4}$ Department of Psychosis Studies, Institute of Psychiatry Psychology and Neuroscience, King's College London, London, UK;

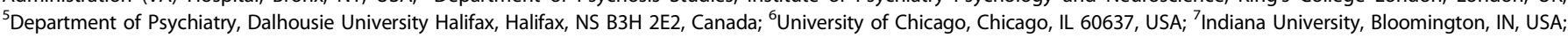
${ }^{8}$ Center for Neurobiology and Behavior, University of Pennsylvania Perelman School of Medicine, Philadelphia, PA, USA; ${ }^{9}$ University of Michigan, Ann Arbor, MI, USA; ${ }^{10}$ Department of Psychiatry, Case Western Reserve University, Cleveland, OH 44106, USA; ${ }^{11}$ University of lowa, lowa City, IA, USA; ${ }^{2}$ University of California San Diego, San Diego, CA, USA; ${ }^{13}$ Mayo Clinic, Rochester, MN, USA; ${ }^{14}$ Department of Psychiatry, Johns Hopkins School of Medicine, Baltimore, MD, USA; ${ }^{15}$ NORMENT, Division of Psychiatry, Haukeland University Hospital and Department of Clinical Medicine, University of Bergen, Bergen, Norway; ${ }^{16}$ Department of Addiction Medicine, Haukeland University Hospital, University of Bergen, Bergen,

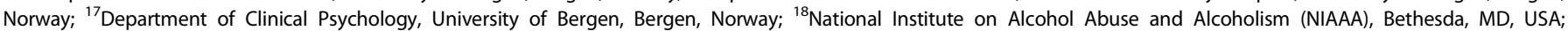
${ }^{19}$ Department of Psychiatry, Stavanger University Hospital, Stavanger, Norway and ${ }^{20}$ Centre for Addiction and Mental Health, University of Toronto, Toronto, ON, Canada Correspondence: Katherine E. Burdick (Kburdick1@bwh.harvard.edu)
}

Received: 1 February 2020 Revised: 24 March 2020 Accepted: 17 April 2020 Published online: 29 April 2020 


\section{4}

manifested by mental slowness, as the side effect most likely to precipitate lithium nonadherence [16]. While BD patients' own perception of their cognitive abilities has been demonstrated to be relatively discordant with objective measures of neurocognitive functioning [17], these perceived side effects may nonetheless result in lithium non-compliance and ultimately mood destabilization. There is a surprising dearth of controlled data evaluating the objective cognitive side effects of lithium treatment in BD.

A relatively recent meta-analysis by Wingo et al. [18] summarized the existing literature on the cognitive effects of lithium, which to date included a total of 213 healthy controls and 326 affective disorder patients (73\% BD; 19.6\% recurrent unipolar; $7.4 \%$ cycloid of schizoaffective). The on lithium group consisted of 276 individuals (105 healthy volunteers; 171 affective disorder patients) versus 263 individuals who were not taking lithium (108 healthy volunteers; 155 affective disorder patients) [18]. When analyzed together, those subjects taking lithium showed minor deficits on verbal learning and creativity as compared with those not taking lithium. When grouped by diagnostic status, the healthy controls exposed to short-term lithium treatment (mean duration of 2.5 weeks) did not differ from healthy subjects who were not exposed to lithium. In contrast, affective disorder patients on long-term lithium treatment (mean duration of 47 months) showed mild deficits on verbal learning and creativity and moderate impairments on psychomotor speed relative to patients not taking lithium [18]. The authors concluded that lithium use over a longer duration is associated with mild neurocognitive deficits; however, several limitations to interpreting these data were acknowledged including a lack of information on relevant illness parameters (e.g. number of prior manias; history of psychosis; and importantly concomitant psychotropic medications). In addition to these limitations, prior studies of lithium and cognition have generally not accounted for dosage and plasma lithium levels, treatment parameters that may be relevant to cognitive outcome.

Despite the results of this single meta-analysis, data on cognitive outcomes after lithium exposure are equivocal and there remains a lack of clarity around lithium's exact mechanism of action [19]. Indeed, many of the known intracellular and molecular effects of lithium are suggestive of beneficial effects on brain structure and function [19], which should intuitively result in pro-cognitive effects. There is in fact some evidence to support a neuroprotective role for lithium in reducing the incidence of dementia in patients with BD [20], as well as some early longitudinal data that evidence no clear cognitive change over time in BD patients treated with lithium [21, 22].

In the current study, we systematically tested the effects of lithium treatment on neurocognitive functioning in patients with $\mathrm{BD}$ in the largest single cohort reported to date by harnessing the power of a multi-site, ongoing pharmacogenetic trial of lithium monotherapy. We first assessed the cognitive effects of lithium treatment cross-sectionally in $262 \mathrm{BD}$ subjects and then followed a subset of these patients $(n=88)$ who achieved mood stabilization after being optimized on lithium monotherapy, allowing for longitudinal analyses of this cohort.

\section{MATERIALS AND METHODS}

This study is an 11-site, prospective, non-randomized, open trial of lithium designed to collect a very large cohort of patients with bipolar I disorder to be treated with lithium monotherapy to promote relapse prevention. The primary purpose of this study was to identify pharmacogenetic predictors of lithium response using genome-wide association study. Several biological and clinical predictors of treatment response are included in the design. Here, we are focusing on the neurocognitive data collected in the context of this trial. A full description of the methods of this trial can be found in Oedegaard et al. [23]. The study was conducted in accordance with the Declaration of Helsinki and the protocol was approved by local IRBs at all sites in the USA and Canada, as well as the Ethics Committee and Health Authorities in Norway. All subjects provided written informed consent prior to their inclusion in the study.

Briefly, patients were systematically diagnosed using the diagnostic interview for genetic studies [24]. Each patient was categorized at baseline with regard to prior lithium exposure into one of four groups: (1) clinically stable (see details below) patients who were currently taking lithium monotherapy (current lithium monotherapy patients- CLM); (2) clinically stable patients who were currently taking lithium plus some additional psychotropic medication(s) (current lithium polytherapy patients- CLP); (3) patients who were currently taking lithium but were not clinically stable (current lithium but unstable patients-CLU); and (4) patients who were not taking lithium (not currently on lithium patients-NCL). Neurocognitive testing was completed for the full sample at baseline. Time 2 testing was only completed for a subset of the subjects who entered the maintenance phase of the study (the exact timing of which varied dependent upon clinical response parameters). Specifically, after lithium monotherapy treatment was initiated at the baseline visit, dosing was optimized over a variable timeframe (up to 3 months of open stabilization phase) until the patient achieved a rating of 1 (normal, not ill), or 2 (minimally ill) on the Clinical Global Impressions of Severity Scale for Bipolar Disorder (CGI-S-BP Overall Bipolar Illness) for 4 out of 5 preceding weeks (observation phase). Once this milestone was achieved, cognitive testing was repeated and patients entered a 2year follow-up period on lithium monotherapy. Subjects who did not respond adequately to lithium monotherapy were treated as per protocol (e.g., dropped from the study, switched to valproate or switched to treatment as usual) but were not tested at a second time point.

\section{Clinical and neurocognitive measures}

To evaluate current severity of mood symptoms clinicianadministered and self-report measures were used; the Clinician Administered Rating Scale for Mania (CARS-M [25]) and the Montgomery Asberg Depression Rating Scale (MADRS [26]); the Quick Inventory of Depressive Symptomatology Self-Report (QIDSSR 16 [27]) was completed at each visit. Mood ratings were conducted at each visit to account for current symptom severity and quality of life self-reports were also completed.

Neurocognitive performance was assessed using the following tasks: Trail-Making Test-part A (TMT-A; processing speed), Trail Making Test-part B (TMT-B; executive function and set-shifting); WAIS-III Digit Symbol subtest (visual scanning and psychomotor speed); Controlled Oral Word Association Test (COWAT; phonemic fluency); California Verbal Learning Test (CVLT) Trials 1-5 total and CVLT delayed recall (verbal learning and memory). For a detailed description of the cognitive measures please refer to Strauss et al. [28]. An estimate of premorbid IQ was obtained using the Wide Range Achievement Test-3rd edition-Reading subtest (WRAT-3; [29]). Raw scores from each measure were used in analyses but $Z$ scores were calculated using the full BD sample (within sample $Z$ scores) for purposes of graphic representation on a uniform scale and in order to provide a basis for calculating the global cognition index.

A global cognitive index was obtained by calculating an unweighted average of all of the $Z$ scores from the individual cognitive measures.

Statistical analysis

Cross-sectional analysis. Cross-sectional analyses focused on lithium status at baseline. We first compared subjects that were on lithium at baseline ( $\mathrm{L} \mathbf{i}+$ yes) versus those subjects that were not on lithium ( $\mathrm{L}+\mathrm{+} \mathrm{no})$ across a range of clinical and demographic 
characteristics using analysis of variance (ANOVA) or Chi-square where appropriate. A series of univariate analyses was conducted (covarying for age and current depressive and manic symptoms as measured by the MADRS and CARS-M respectively) to examine potential group differences across each of the cognitive tests individually. This was done instead of a multivariate ANOVA to optimize the number of subjects included in each analysis (due to scattered missing datapoints on different measures). The next level of cross-sectional analyses focused on the more specific subgrouping of the patients at baseline. As noted above, patients were categorized as follows at baseline: (1) clinically stable currently taking lithium monotherapy (CLM); (2) clinically stable currently taking lithium polytherapy (CLP); (3) those taking lithium but clinically unstable (CLU); and (4) those who were not currently taking lithium (NCL). These four groups were compared in terms of clinical, demographic, and cognitive characteristics using the same approach as above

Next, using the prospective sample of patients who were optimized on lithium monotherapy as part of this study, we began to address several of these limitations using longitudinal analyses.

Prospective (longitudinal) analysis

A series of repeated measures analysis of covariance (ANCOVA) models were used to explore the effect of lithium treatment across time for each of the neurocognitive tests. We did not include a between-subjects fixed factor, as all patients were treated with lithium monotherapy; cognitive performance (CVLT trial 1-5, CVLT delayed recall, Digit Symbol, TMT-A, TMT-B, and a global cognitive index) at baseline and at follow-up served as the within-subjects variables. Covariates included affective change scores (differences between depressive and manic symptoms at follow-up and at baseline). Sensitivity analyses tested whether plasma lithium levels at follow-up contributed to cognitive change.

In addition to repeated measures analyses in an effort to account for the lack of a placebo arm and to estimate reliable change over time, we calculated a reliable change index $(\mathrm{RCl})$ for cognitive measures showing evidence for change in ANCOVA models models [30]. This approach allows for the evaluation of whether a change over time in an individual's score (e.g., baseline versus follow-up) is considered statistically significant. The $\mathrm{RCl}$ is represented as a ratio, the numerator is the observed change and the denominator is a standard measure of the expected difference. Cognitive tests have the advantage of having good reliability data, which can be used as the denominator in $\mathrm{RCl}$ analyses. We selected the CVLT for $\mathrm{RCl}$ analyses in this study, as it has substantial reliability data published on it. We could not easily do the same for the global cognitive index but expect based upon the consistency of the findings that we would have found similar estimates of improvement at the individual level. As it is customary using the $\mathrm{RCl}$, we report the percent of patients that showed reliable improvement, those who showed reliable deterioration, and those who showed no reliable change.

\section{RESULTS}

Cross-sectional results

Full sample descriptive at baseline. Two hundred sixty-two BD I patients entered the study; 122 were males (45.9\%) and the mean age was 42.7 years $(S D=14.5)$. At baseline, there were no clinically relevant manic symptoms as assessed by the CARS-M (mean $=5.4$; $\mathrm{SD}= \pm 7.0$ ) while some mild depressive symptoms were noted on the MADRS (mean $=11.9 ; \mathrm{SD}= \pm 11.4$ ).

Lithium yes/no at baseline. Of the 262 subjects tested at baseline, 169 were taking lithium (Li/yes) and 93 were not on lithium (Li/no). There were statistically significant clinical and demographic differences when comparing BD patients who were on lithium with those who were not on lithium at baseline (Table 1). Subjects that were not taking lithium were younger $(p=0.002)$, less likely to be white $(p<0.001)$ and had more severe current manic and

Table 1. Group baseline comparisons between patients that were on lithium ( $\mathrm{Li}+\mathrm{yes}$ ) and those who were not on lithium ( $\mathrm{Li}+\mathrm{no}$ ).

\begin{tabular}{|c|c|c|c|c|}
\hline Variable name & $\begin{array}{l}\mathrm{Li}+\mathrm{no} \\
(n=93)\end{array}$ & $\begin{array}{l}\text { Li+yes } \\
(n=169)\end{array}$ & $\begin{array}{l}\text { Test statistic } \\
\mathrm{X}^{2} / \mathrm{ANOVA} \text { (df) }\end{array}$ & $P$ value \\
\hline & $N(\%)$ & $N(\%)$ & & \\
\hline Male & $45(48.4)$ & $76(45.0)$ & $X^{2}(1)=0.3$ & 0.6 \\
\hline Female & 48 (51.6) & $93(55.0)$ & & \\
\hline \multirow[t]{2}{*}{ Non-white } & $27(29.0)$ & 19 (11.2) & & \\
\hline & Mean (SD) & Mean (SD) & & \\
\hline Age & $38.9(12.1)$ & $44.7(15.3)$ & $F(1,264)=10.0$ & 0.002 \\
\hline Manic symptoms (CARS-M) & $8.2(6.4)$ & $3.9(6.9)$ & $F(1,256)=24.7$ & $<0.001$ \\
\hline Depressive symptoms (MADRS) & $19.7(11.5)$ & $7.6(8.8)$ & $F(1,261)=90.3$ & $<0.001$ \\
\hline Digit symbol & $63.0(17.1)$ & $60.4(16.8)$ & $F(1,260)=1.4$ & 0.2 \\
\hline WRAT & $103.1(13.6)$ & $105.7(10.5)$ & $F(1,241)=2.7$ & 0.1 \\
\hline COWAT & $38.3(10.8)$ & $39.3(11.6)$ & $F(1,257)=0.5$ & 0.5 \\
\hline TMT-A & $30.2(13.3)$ & $32.8(12.5)$ & $F(1,258)=2.5$ & 0.1 \\
\hline TMT-B & $80.1(41)$ & 81.5 (41.6) & $F(1,254)=0.07$ & 0.8 \\
\hline
\end{tabular}




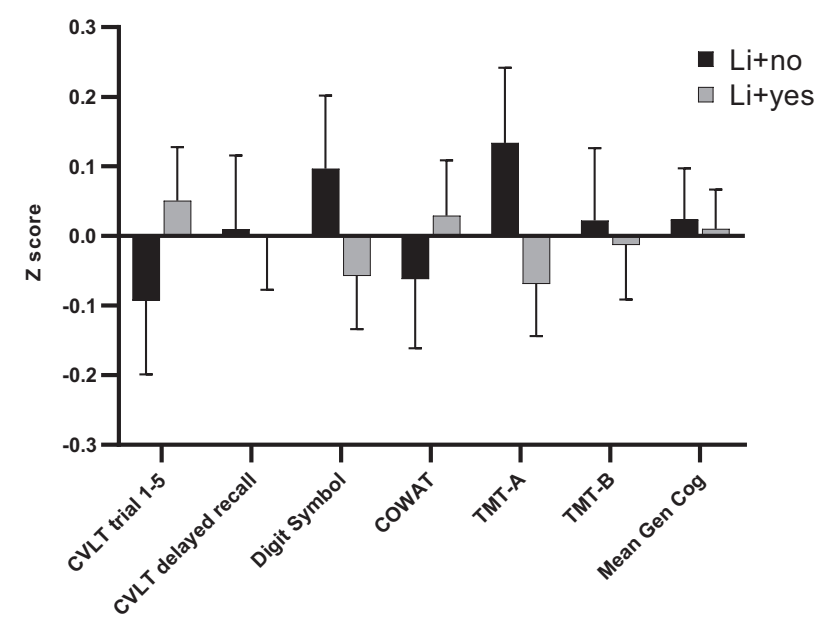

Fig. 1 Cognitive performance at baseline of bipolar disorder patients taking lithium $(N=169)$ vs. those not taking lithium $(N=$ 93) were compared across six neurocognitive tests (CVLT trials 1-5, CVLT delayed recall, Digit Symbol, COWAT, TMT-A, and TMTB) and a global cognitive index. Scores are expressed as $Z$ scores with a mean of 0 and standard deviation of 1 and were calculated within the BD sample. Error bars are depicted to describe variability of the data.

depressive symptoms (both clinician-assessed and self-reported) than those subjects taking lithium (both significant at $p<0.001$; Table 1). Using these variables as covariates, when comparing Li/ yes vs. Li/no subjects on neurocognition, we found no significant group differences for either the global cognitive index any of the or individual cognitive tests (Table 1). When controlling for age, race, and mood state (CARS-M and MADRS scales) there were no group differences between Li/yes and Li/no groups. Cognitive performance of the two groups is depicted in Fig. 1.

Four lithium subgroups at baseline. Of the 262 subjects tested at baseline, 73 were on monotherapy (CLM), 44 were on polytherapy (CLP), 52 were currently on lithium but clinically unstable (CLU), and 93 were not currently taking lithium (NCL). The cognitive profiles of these four groups are depicted in Fig. 2. When comparing neurocognitive performance across groups, we found a significant effect of group on CVLT trials $1-5[F(3,254)=3.370$, $p=0.019]$. Post hoc comparisons revealed that the CLM subjects performed significantly better than all other groups on CVLT trials $1-5$ : CLP subjects (mean difference $=0.53, \mathrm{SE}=0.19 ; p=0.005$ ); $\mathrm{CLU}$ subjects (mean difference $=0.37, \mathrm{SE}=0.18 ; p=0.04$ ); $\mathrm{NCL}$ subjects (mean difference $=0.39, \mathrm{SE}=0.16 ; p=0.01$ ).

Prospective results

Eighty-eight BD I patients, baseline status (CLM N=22, CLP $N=$ $14, \mathrm{CLU} N=21, \mathrm{NCL} N=31$ ), completed data at both baseline and, after the initiation of lithium monotherapy, at the time that the patient entered the maintenance phase of the study. The mean age of these patients was $40.9(\mathrm{SD}= \pm 14.2), 37$ were male $(42 \%)$, $69(78.4 \%)$ were white; and the average premorbid IQ was 103.1 $(\mathrm{SD}= \pm 13.9)$. At baseline, the mean CARS-M score was $5.7(\mathrm{SD}=$ \pm 8.9 ), which indicates an absence of clinically significant manic symptoms; the mean MADRS score was $10.5(\mathrm{SD}= \pm 10.3)$ which falls within the range indicative of mild depression. To determine the representativeness of our prospective sample, we compared it to the full cohort using baseline data. There were no statistically significant differences between the two groups in terms of demographic, clinical or cognitive characteristics.

The average daily dosage of lithium at the time of the first neurocognitive assessment (in the subset of patients already

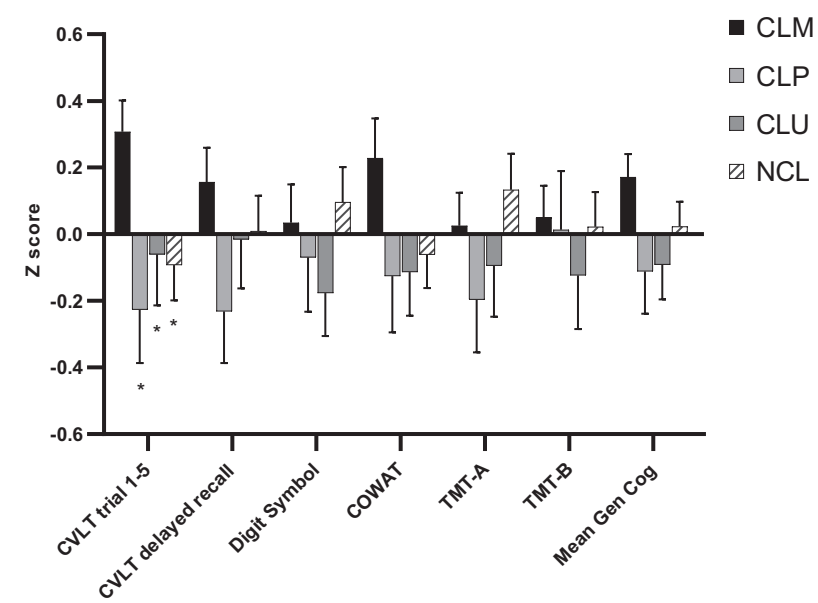

Fig. 2 Performance across six cognitive tests and global cognitive index was compared across bipolar disorder patients grouped according their lithium treatment regimen at baseline. a Not currently on lithium ( $\mathrm{NCL} ; N=93)$. b Currently on lithium but unstable (CLU; $N=52)$. c Currently on lithium polytherapy (CLP; $N=$ 44). d Currently on lithium monotherapy (CLM; $N=73$ ). $Z$ scores (with mean of zero and standard deviation of 1) are used. Error bars are depicted to describe variability of the data.

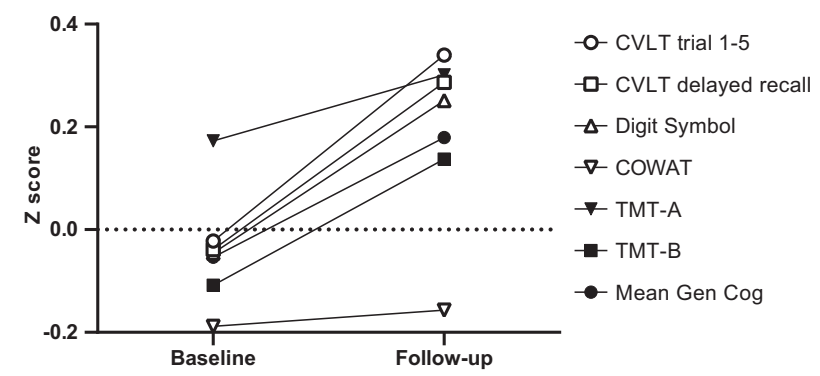

Fig. 3 Change in performance (of a subset of the entire cohort) from baseline to follow-up on the six neurocognitive tests (CVLT trials 1-5, CVLT delayed recall, Digit Symbol, COWAT, TMT-A, and TMT-B) and a global cognitive index in BD subjects taking lithium is reported. $Z$ scores (with mean of 1 and standard deviation of 1 ) and calculated within the BD sample are used. ${ }^{*} p<0.05$.

taking lithium at entry) was $914.4 \mathrm{mg}(\mathrm{SD}= \pm 365.3)$ and the mean plasma lithium levels at baseline in the same subset was 0.63 $(\mathrm{SD}=0.27$; range 0.1-2.2). At follow-up, the mean dosage was $929.2 \mathrm{mg}(\mathrm{SD}= \pm 356.0)$ and mean plasma lithium levels were 0.65 $(S D=0.22$ range $0.1-1.4)$. The average duration between the first and the second neurocognitive assessment was 132.1 days (SD = \pm 90.3 ) with a wide range (32 days to 485 days). This variability was due to the inter-individual differences in time to reach stabilization criteria.

As expected, clinical improvement was noted on mania $(5.7 \pm$ 8.9 vs. $1.8 \pm 3.4$, respectively at baseline and follow-up) and depression symptom scales $(10.5 \pm 10.3$ vs. $4.7 \pm 5.4)$; therefore, these were included as covariates in RMANOVAs. When comparing cognitive performance at baseline vs. follow-up, analyses revealed significant neurocognitive improvement in the global cognitive index score $[F=31.69 ; p<0.001]$, CVLT trials $1-5[F=29.81 ; p<$ $0.001]$, CVLT delayed recall $[F=15.27 ; p<0.001]$, and TMT-B [ $F=$ $6.64, p=0.012$ ] (Fig. 3). None of the cognitive measures evidenced decline over time with lithium treatment. When plasma lithium levels drawn at follow-up were added as covariates, the main 
effect of level was non-significant for all analyses and cognitive results remained significant.

Analyses calculating the $\mathrm{RCl}$ were carried out for the CVLT indicating that $30.7 \%(27 / 88)$ of patients showed a reliable improvement on this task over time; $63.6 \%(56 / 88)$ of patients showed no significant change; and only $5.7 \%(5 / 88)$ showed evidence of reliable deterioration.

There were non-significant correlations between plasma lithium levels and cognition (global cognitive index) at baseline (in the subset taking lithium at entry; $r=-0.109 ; p=0.195$ ) or at followup $(r=0.053 ; p=0.648)$.

\section{DISCUSSION}

Lithium remains one of the most effective treatments for reducing relapse and preventing suicide in patients with $\mathrm{BD}$. There are several side effects that have limited its use by clinicians; primarily adverse effects on renal and endocrine functioning. A very common complaint noted by patients who are taking lithium is related to a subjective sense of neurocognitive dulling, which is often a trigger for treatment non-compliance. Despite substantial anecdotal evidence, very few systematic studies have addressed the cognitive side effects of lithium. Our study is not the first to do so; however, we have the advantage of the largest cross-sectional cohort collected to date with cognitive measures and lithium treatment status. Moreover, we tracked a relatively large subset of this sample as they transitioned from various treatment regimens to lithium monotherapy with longitudinal data available to address cognitive changes.

Our results from both cross-sectional and longitudinal analyses broadly suggest that lithium treatment does not significantly impair neurocognitive functions in patients with BD. Indeed, crosssectional analyses comparing 169 patients currently taking lithium with 93 patients not taking lithium did not reveal any significant group differences on neurocognitive tests (CVLT trials 1-5, CVLT delayed recall, Digit Symbol, TMT-A, and TMT-B) or on global cognition-even after controlling for demographics and current mood symptoms. We were also able to analyze longitudinal data from a subset of this cohort who received follow-up cognitive testing after being stabilized on lithium monotherapy. This was not a placebo-controlled trial so practice effects on cognitive measure cannot be fully controlled for; however, performance across all cognitive tests improved over the course of treatment and $\mathrm{RCl}$ indices suggest a much larger proportion of patients improved $(\sim 31 \%)$ than would be expected $(\sim 3 \%)$ to reliably improve based upon the psychometrics of the measures. These proportions are contrasted with what has been reported in healthy individuals on the CVLT with a one-month lag in repeat testing [31], where only $\sim 3 \%$ of individuals show reliable improvement and $\sim 96 \%$ show no change. Although we cannot interpret this as a cognitive benefit, these data do suggest that lithium did not result in deleterious effects on cognition.

These findings are largely consistent with early small-scale studies that reported no significant negative effects of lithium on memory functions over a 4 and 12-month follow-up [22], as well as over a 6-year interval [21]. More recent meta-analyses note mild impairments on measures of processing speed, verbal learning, and creativity in bipolar subjects who had been exposed to longterm lithium treatment (mean of 47 months); however, this was based solely upon cross-sectional comparisons with subjects not taking lithium and many of the participants were taking other psychotropic medications. In the same analysis, healthy subjects exposed to lithium over a shorter duration did not evidence any cognitive impairment.

The exact mechanism of action that makes lithium an effective mood stabilizer is unknown; however, there is mounting evidence that it has neuroprotective effects [32] which may be related to lithium's effects on neuronal homeostasis [33]. In preclinical studies of transgenic mice that overexpress GSK-3 $\beta$, chronic lithium treatment can reverse neuropathology associated with Alzheimer's disease (tau phosphorylation and $A \beta$ production) and improve memory deficits [34-37] via direct modulation of APP and inhibition of GSK-3 $\beta[38,39]$. In addition, lithium has been shown to stimulate the expression of anti-apoptotic proteins (e.g., $\mathrm{Bcl}-2$ ) promoting neuronal survival [40] and stimulate the synthesis of neurotrophic factors such as brain-derived neurotrophic factor (BDNF) and vascular endothelial growth factor to promote hippocampal neurogenesis, synaptic plasticity, and long-term potentiation [41]. Lithium also serves to modulate inflammatory processes by reducing pro-inflammatory cytokines and microglial activation in animal models of stroke [42]. Convergent data from clinical and neuroimaging studies support the myriad of beneficial effects seen at the cellular level. Registry data suggest that bipolar patients chronically treated with lithium are significantly less likely to develop dementia than patients taking other mood stabilizers or not taking medications $[20,43]$. Antioxidant and pro-BDNF effects have also been linked with lithium treatment response in BD patients [44] and in healthy subjects [45]. Neuroimaging studies support lithium's neuroprotective effect, with data suggesting that long-term lithium treatment is associated with increased volume of the hippocampus and amygdala and increased cortical thickness [46-50]. Taken together, data from preclinical, clinical, and neuroimaging-based studies strongly support lithium's role in neuroprotection and our data are consistent with these findings.

Overall, data derived from our large cohort of BD patients taking lithium suggest no deleterious effects on neurocognitive functioning, despite the frequent subjective complaints of cognitive dulling noted by patients treated with this agent. It might be speculated that the subjective self-reports of cognitive impairment after starting lithium are related more directly to stabilization of affective symptoms that may be deemed as positive (e.g., hypomania) to the patient. There are some limitations to our work including the lack of a placebo-control; however, this was a realworld trial with a focus on real-world treatment response. The neurocognitive battery was by design very brief to allow for across site data collection; therefore, we did not assay broader domains such as creativity and we cannot draw any conclusions outside of the core cognitive tests assessed. Although to our knowledge this is the largest cohort of BD patients with both information on lithium treatment and cognitive data, our follow-up longitudinal analyses focused on lithium monotherapy were carried out in a relatively small sample $(n=88)$. Most critically, we did not have cognitive testing available at a second time-point in those patients who did not clinically respond to lithium, so we are only able to comment on lithium's effects in lithium responders. Larger-scale, controlled trials are warranted to confirm our results.

There is ample evidence from prior work that lithium acts in the brain in a largely beneficial manner to protect neurons from toxicity and to promote new cell growth. Anecdotal evidence has suggested counterintuitive deleterious effects on cognition; however, very few systematic studies have been done to directly address this. Our data from cross-sectional and longitudinal analyses suggest that lithium may be beneficial to neurocognitive functioning in patients with BD and that at the very least it does not seem to significantly impair cognition when used therapeutically. This may have important implications to clinicians as they discuss with their patients the pros and cons of this agent in mood stabilization.

\section{FUNDING AND DISCLOSURE}

This work was supported by the US National Institutes of Mental Health and General Medical Sciences (MH92758 to JRK; MH100125 to KEB). KEB has served as an advisory board member to Dainippon Sumitomo Pharmaceutical Neuralstem, and Takeda- 
Lundbeck. JN has received funding as an investigator from Assurex and from Janssen. JRK has received funds from Pathway Genomics as an investigator. All other authors have nothing to disclose.

\section{ACKNOWLEDGEMENTS}

The authors thank the patient participants without whom this work would not be possible.

\section{AUTHOR CONTRIBUTIONS}

JRK organized the consortium from which the data were generated. KEB conceived of the presented idea. KEB, CEM and MR participated in data analysis, interpretation of results, as well as the writing and critical review of the manuscript. $M A, N A, A A, Y B$, WB, HB, JRC, C Calkin, C Conroy, WC, AD, SF, CF, NF, MF, KG, JG, ESG, KG, FSG, TG, GM, GJH, PJ, M Kamali, M Kelly, SL, EML, FWL, AXM, MJM, MM, CMN, JN, KJO, AO, MR, KR, M Schinagle, CS, M Shaw, PS, CS, ES, BT, and PZ assisted in data collection and review of the manuscript.

\section{ADDITIONAL INFORMATION}

Publisher's note Springer Nature remains neutral with regard to jurisdictional claims in published maps and institutional affiliations.

\section{REFERENCES}

1. Burdick EK, Goldberg ET, Cornblatt AB, Keefe SR, Gopin BC, Derosse P, et al. The MATRICS consensus cognitive battery in patients with bipolar I disorder. Neuropsychopharmacology. 2011;36:1587-92.

2. Bora $E$, Yucel $M$, Pantelis C. Cognitive endophenotypes of bipolar disorder: a meta-analysis of neuropsychological deficits in euthymic patients and their firstdegree relatives. J Affect Disord. 2009;113:1-20.

3. Burdick KE, Goldberg JF, Harrow M, Faull RN, Malhotra AK. Neurocognition as a stable endophenotype in bipolar disorder and schizophrenia. J Nerv Ment Dis. 2006;194:255-60.

4. Depp CA, Mausbach BT, Harmell AL, Savla GN, Bowie CR, Harvey PD, et al. Metaanalysis of the association between cognitive abilities and everyday functioning in bipolar disorder. Bipolar Disord. 2012;14:217-26.

5. Burdick EK, Russo M, Frangou S, Mahon K, Braga JR, Shanahan M, et al. Empirical evidence for discrete neurocognitive subgroups in bipolar disorder: clinical implications. Psychol Med. 2014;44:3083-96.

6. Bora E, Pantelis C. Meta-analysis of cognitive impairment in first-episode bipolar disorder: Comparison with first-episode schizophrenia and healthy controls. Schizophr Bull. 2015;41:1095-104.

7. Glahn DC, Almasy L, Barguil M, Hare E, Peralta JM, Kent JW, et al. Neurocognitive endophenotypes for bipolar disorder identified in multiplex multigenerational families. Arch Gen Psychiatry. 2010;67:168-77.

8. Naiberg MR, Newton DF, Collins JE, Dickstein DP, Bowie CR, Goldstein BI. Elevated triglycerides are associated with decreased executive function among adolescents with bipolar disorder. Acta Psychiatr Scand. 2016;134:241-8.

9. Naiberg MR, Newton DF, Collins JE, Bowie CR, Goldstein BI. Impulsivity is associated with blood pressure and waist circumference among adolescents with bipolar disorder. J Psychiatr Res. 2016;83:230-9.

10. Czepielewski LS, Massuda R, Goi P, Sulzbach-Vianna M, Reckziegel R, Costanzi M, et al. Verbal episodic memory along the course of schizophrenia and bipolar disorder: a new perspective. Eur Neuropsychopharmacol. 2015;25:169-75.

11. Geddes JR, Burgess S, Hawton K, Jamison K, Goodwin GM. Long-term lithium therapy for bipolar disorder: systematic review and meta-analysis of randomized controlled trials. Am J Psychiatry. 2004;161:217-22.

12. Geddes JR, Miklowitz DJ. Treatment of bipolar disorder. Lancet. 2013;381:1672-82.

13. Shine B, Mcknight RF, Leaver L, Geddes JR. Long-term effects of lithium on renal, thyroid, and parathyroid function: A retrospective analysis of laboratory data. Lancet. 2015;386:461-8.

14. Grandjean EM, Aubry J-M. Lithium: updated human knowledge using an evidence-based approach. Part II: Clinical pharmacology and therapeutic monitoring. CNS Drugs. 2009;23:331-49.

15. Pachet AK, Wisniewski AM. The effects of lithium on cognition: an updated review. Psychopharmacology. 2003;170:225-34.

16. Gitlin M. Lithium side effects and toxicity: prevalence and management strategies. Int J Bipolar Disord. 2016;4:27.
17. Burdick KE, Endick CJ, Goldberg JF. Assessing cognitive deficits in bipolar disorder: are self-reports valid? Psychiatry Res. 2005;136:43-50.

18. Wingo AP, Wingo TS, Harvey PD, Baldessarini RJ. Effects of lithium on cognitive performance: a meta-analysis. J Clin Psychiatry. 2009;70:1588-97.

19. Malhi GS, Tanious M, Das P, Coulston CM, Berk M. Potential mechanisms of action of lithium in bipolar disorder: Current understanding. CNS Drugs. 2013;27: 135-53.

20. Kessing LV, Forman JL, Andersen PK. Does lithium protect against dementia? Bipolar Disord. 2010;12:87-94.

21. Engelsmann F, Katz J, Ghadirian AM, Schachter D. Lithium and memory: a longterm follow-up study. J Clin Psychopharmacol. 1988;8:207-12.

22. Smigan L, Perris C. Memory functions and prophylactic treatment with lithium. Psychol Med. 1983;13:529-36.

23. Oedegaard JK, Alda M, Anand A, Andreassen AO, Balaraman Y, Berrettini HW, et al. The Pharmacogenomics of Bipolar Disorder study (PGBD): identification of genes for lithium response in a prospective sample. BMC Psychiatry. 2016;16:129. https://doi.org/10.1186/s12888-016-0732-x.

24. Nurnberger JI Jr, Blehar MC, Kaufmann CA, York-Cooler C, Simpson SG, HarkavyFriedman J, et al. Diagnostic interview for genetic studies: rationale, unique features, and training. NIMH Genetics Initiative. Arch Gen Psychiatry. 1994;51:849-59; discussion 863-4. PubMed PMID: 7944874.

25. Altman EG, Hedeker DR, Janicak PG, Peterson JL, Davis JM. The clinicianadministered rating scale for mania (CARS-M): Development, reliability, and validity. Biol Psychiatry. 1994;36:124-34.

26. Montgomery SA, Asberg M. A new depression scale designed to be sensitive to change. Br J Psychiatry. 1979;134:382-9.

27. Rush AJ, Trivedi MH, Ibrahim HM, Carmody TJ, Arnow B, Klein DN, et al. The 16item Quick Inventory of Depressive Symptomatology (QIDS), clinician rating (QIDS-C), and self-report (QIDS-SR): A psychometric evaluation in patients with chronic major depression. Biol Psychiatry. 2003;54:573-83.

28. Strauss E, Sherman E, Spreen O. A compendium of of neuropsychological tests: administration, norms and commentary. USA: Oxford University Press; 2006.

29. Wilkinson GS. Wide range achievement test 3. Wilmington, DE: Wide Range, Inc.; 1993.

30. Guhn M, Forer B, Zumbo BD. Reliable change index. In: Michalos AC, editor. Encyclopedia of quality of life and well-being research, Dordrecht: Springer Netherlands; 2014. p. 5459-62.

31. Woods SP, Delis DC, Scott JC, Kramer JH, Holdnack JA. The California Verbal Learning Test-second edition: test-retest reliability, practice effects, and reliable change indices for the standard and alternate forms. Arch Clin Neuropsychol. 2006;21:413-20.

32. Forlenza OV, De-Paula VJR, Diniz BSO. Neuroprotective effects of lithium: Implications for the treatment of Alzheimer's disease and related neurodegenerative disorders. ACS Chem Neurosci. 2014;5:443-50.

33. Machado-Vieira R, Manji HK, Zarate CA. The role of lithium in the treatment of bipolar disorder: Convergent evidence for neurotrophic effects as a unifying hypothesis. Bipolar Disord. 2009;11:92-109.

34. Engel T, Goñi-Oliver P, Lucas JJ, Avila J, Hernández F. Chronic lithium administration to FTDP-17 tau and GSK-3 $\beta$ overexpressing mice prevents tau hyperphosphorylation and neurofibrillary tangle formation, but pre-formed neurofibrillary tangles do not revert. J Neurochem. 2006;99:1445-55.

35. Leroy K, Ando K, Héraud C, Yilmaz Z, Authelet M, Boeynaems JM, et al. Lithium treatment arrests the development of neurofibrillary tangles in mutant tau transgenic mice with advanced neurofibrillary pathology. J Alzheimers Dis. 2010;19:705-19.

36. Noble W, Planel E, Zehr C, Olm V, Meyerson J, Suleman F, et al. Inhibition of glycogen synthase kinase- 3 by lithium correlates with reduced tauopathy and degeneration in vivo. Proc Natl Acad Sci USA. 2005;102:6990-5.

37. Matsunaga S, Kishi T, Annas P, Basun H, Hampel H, Iwata N. Lithium as a treatment for Alzheimer's disease: a systematic review and meta-analysis. J Alzheimers Dis. 2015;48:403-10.

38. Rockenstein E, Torrance M, Adame A, Mante M, Bar-on P, Rose JB, et al. Neuroprotective effects of regulators of the glycogen synthase kinase-3 $\beta$ signaling pathway in a transgenic model of Alzheimer's disease are associated with reduced amyloid precursor protein phosphorylation. J Neurosci. 2007;27: 1981-91.

39. Su Y, Ryder J, Li B, Wu X, Fox N, Solenberg $P$, et al. Lithium, a common drug for bipolar disorder treatment, regulates amyloid- $\beta$ precursor protein processing. Biochemistry. 2004;43:6899-908.

40. Chen RW, Chuang DM. Long term lithium treatment suppresses p53 and Bax expression but increases $\mathrm{Bcl}-2$ expression. A prominent role in neuroprotection against excitotoxicity. J Biol Chem. 1999;274:6039-42.

41. Diniz BS, Teixeira AL. Brain-derived neurotrophic factor and Alzheimer's disease: Physiopathology and beyond. NeuroMolecular Med. 2011;13:217-22. 
The association between lithium use and neurocognitive performance in... KE Burdick et al.

42. Li H, Li Q, Du X, Sun Y, Wang X, Kroemer G, et al. Lithium-mediated long-term neuroprotection in neonatal rat hypoxia-ischemia is associated with antiinflammatory effects and enhanced proliferation and survival of neural stem/progenitor cells. J Cereb Blood Flow Metab. 2011;31:2106-15.

43. Kessing LV, Søndergård L, Forman JL, Andersen PK. Lithium treatment and risk of dementia. Arch Gen Psychiatry. 2008;65:1331-5.

44. de Sousa RT, van de Bilt MT, Diniz BS, Ladeira RB, Portela LV, Souza DO, et al. Lithium increases plasma brain-derived neurotrophic factor in acute bipolar mania: a preliminary 4-week study. Neurosci Lett. 2011;494:54-56.

45. Khairova R, Pawar R, Salvadore G, Juruena MF, De Sousa RT, Soeiro-De-Souza MG, et al. Effects of lithium on oxidative stress parameters in healthy subjects. Mol Med Rep. 2012;5:680-2.

46. Bearden $C E$, Thompson PM, Dalwani M, Hayashi KM, Lee AD, Nicoletti $M$, et al. Greater cortical gray matter density in lithium-treated patients with bipolar disorder. Biol Psychiatry 2007;62:7-16.
47. van Erp TGM, Thompson PM, Kieseppä T, Bearden CE, Marino AC, Hoftman GD, et al Hippocampal morphology in lithium and non-lithium-treated bipolar I disorder patients, non-bipolar co-twins, and control twins. Hum Brain Mapp. 2012;33:501-10.

48. Lyoo IK, Dager SR, Kim JE, Yoon SJ, Friedman SD, Dunner DL, et al. Lithiuminduced gray matter volume increase as a neural correlate of treatment response in bipolar disorder: a longitudinal brain imaging study. Neuropsychopharmacology. 2010;35:1743-50.

49. Moore GJ, Cortese BM, Glitz DA, Zajac-Benitez C, Quiroz JA, Uhde TW, et al. A longitudinal study of the effects of lithium treatment on prefrontal and subgenual prefrontal gray matter volume in treatment-responsive bipolar disorder patients. J Clin Psychiatry. 2009;70:699-705.

50. Yucel K, McKinnon MC, Taylor VH, Macdonald K, Alda M, Young LT, et al. Bilateral hippocampal volume increases after long-term lithium treatment in patients with bipolar disorder: a longitudinal MRI study. Psychopharmacology. 2007; 195:357-67. 